\title{
Effects of genetic factors on the pharmacokinetics and pharmacodynamics of amlodipine in primary hypertensive patients
}

\author{
CHENGXIAN GUO $^{1}$, QI PEI ${ }^{2}$, HONGYI TAN ${ }^{1}$, ZHIJUN HUANG ${ }^{1}$, HONG YUAN ${ }^{1}$ and GUOPING YANG ${ }^{1}$ \\ ${ }^{1}$ Center of Clinical Pharmacology; ${ }^{2}$ Department of Pharmacy, The Third Xiangya Hospital, \\ Central South University, Changsha, Hunan 410078, P.R. China
}

Received October 24, 2014; Accepted November 14, 2014

DOI: $10.3892 /$ br.2014.395

\begin{abstract}
The aim of the present study was to explore the effects of common genetic polymorphisms of cytochrome P450 (CYP)3A4, CYP3A5, cytochrome P450 oxidoreductase $(P O R)$ and multidrug resistance protein $1(M D R 1)$ on the pharmacokinetics and pharmacodynamics of amlodipine in primary hypertensive patients. The mild-to-moderate essential hypertension patients were recruited to complete the genotyping of $C Y P 3 A 4, C Y P 3 A 5, P O R$ and MDRl by sequencing. After a 1 -week placebo washout period, the subjects received $5 \mathrm{mg}$ oral amlodipine daily for 4 weeks. Serial blood samples were collected prior to the last dosing, and 2, 6 and $24 \mathrm{~h}$ post-dosing. Blood pressures were measured prior and subsequent to dosing, and the demographical data were also collected. The blood samples were collected for laboratory testing. The plasma concentrations of amlodipine were determined by high-performance liquid chromatography/tandem mass spectrometry. A total of 60 patients, including 31 males and 29 females, completed the 4 -week treatment. The plasma concentration of amlodipine in females at each time point was significantly higher compared to males $(\mathrm{P}<0.05)$. However, no significant gender differences existed in antihypertensive efficacy. The genetic polymorphisms of MDRl C3435T had a certain impact on the plasma concentration of amlodipine, but did not affect its antihypertensive efficacy $(\mathrm{P}>0.05)$. The genetic polymorphisms of $C Y P 3 A 4 * 1 G, C Y P 3 A 5 * 3$ and POR A503V showed no impact on plasma concentration and efficacy of amlodipine ( $\mathrm{P}>0.05)$. Gender and $M D R 1$ gene polymorphism may
\end{abstract}

Correspondence to: Professor Guoping Yang, Center of Clinical Pharmacology, The Third Xiangya Hospital, Central South University, 138 Tongzipo Road, Changsha, Hunan 410078, P.R. China

E-mail: ygp9880@126.com

Key words: amlodipine, genetic factors, pharmacokinetics, pharmacodynamics, hypertension affect the plasma concentration of amlodipine in hypertensive patients. However, there was no impact on the efficacy of amlodipine.

\section{Introduction}

As one of the most common cardiovascular diseases, hypertension is an important risk factor for various cardio and cerebrovascular diseases, such as stroke, myocardial infarction, heart failure and chronic kidney disease (1). Previous studies have demonstrated that hypertension may be prevented and is controllable (2-4). Reducing the level of blood pressure in hypertensive patients significantly decreased the occurrences of stroke and heart disease, significantly improved the survival quality of patients and lowered the burden of disease (1).

As a new type of long-acting calcium channel blocker (CCB) within the category of dihydropyridine, amlodipine offers multiple advantages of quick onset, improved efficacies, fewer side-effects and long-term anti-atherosclerotic effects. However, there is no clear effect on blood glucose and lipid metabolism. Thus, it is widely used for treating hypertension.

Evident individual differences exist in curative effect and adverse reactions of amlodipine. Numerous large international multicenter clinical trials have proved that amlodipine could be used as a single agent in the treatment of mild-moderate primary hypertension, with an efficiency $\sim 70 \%(5,6)$. Fogari et al (5) showed that its effective therapeutic rate reached $60 \%$. Fogari et al (5) reported that $20 \%$ of patients had to withdraw its use due to adverse reactions, such as ankle edema, facial flushing and palpitation. Among a number of causative factors for individual differences of amlodipine, genetics may be one of the major ones (6).

Amlodipine is predominantly metabolized by cytochrome P450 family member cytochrome P450 (CYP)3A in liver. In adults, CYP3A consists of CYP3A4 and CYP3A5. The activities of CYP3A may vary greatly among different individuals so as to affect its in vivo pharmacokinetics. As a result, its efficacy or adverse reactions are greatly diverse.

Cytochrome P450 oxidoreductase (POR) acts as a sole electron donor for all liver microsomal CYP450 isozymes $(7,8)$. 
It passes two electrons from NADPH to heme iron in CYP molecules. Electron transfer is the rate-limiting step for CYP-catalyzed oxidation-reduction reaction. The $P O R$ gene polymorphism $(A 503 \mathrm{~V})$ reduced the activity of CYP17, but had no effect on the activities of CYP1A2 and CYP2C19 (9). The experiments of transgenic mice have demonstrated that decreased activity of POR decreased the enzymatic activity of CYP (10). One recent study of Caucasian patients demonstrated that $P O R$ gene mutation $(A 503 \mathrm{~V})$ significantly increased CYP3A activity in vivo (11). The effect of the $P O R$ gene polymorphisms on the activity of CYP3A was greater than that of the CYP3A gene polymorphisms on the activity of CYP3A. Whether the POR gene polymorphism may influence the effect of amlodipine through regulating the activity of CYP3A is unknown.

P-glycoprotein (P-gp) plays an important role in the pharmacokinetics of drugs. Frazier et al (12) found that the plasma drug concentration of amlodipine in healthy volunteers of the MDR1 C3435T mutant allele carrier was lower than that of the CC type. However, according to the study of Cai et al (13), the MDR1 C3435T mutant did not influence the effect of amlodipine in renal transplant patients with hypertension. Therefore, further investigations are required to elucidate the impact of the MDRI C3435T polymorphism on the pharmacokinetics and efficacy of amlodipine.

In the present study, the major objective was to assess whether the common genetic polymorphisms of the $C Y P 3 A 4$, CYP3A5, POR and MDR1 genes affected the pharmacokinetics and pharmacodynamics of amlodipine during the therapy of primary hypertensive patients.

\section{Patients and methods}

Subjects and study design. The present study was approved by the Ethics Committee of the Third Xiangya Hospital, Central South University (Changsha, Hunan, China). The patients of mild-to-moderate essential hypertension were recruited according to the following inclusion criteria: i) Age $\geq 40$ and $\leq 75$ years and male or female; and ii) primary hypertension was diagnosed according to the Chinese Hypertension Prevention Guide 2005 Edition (14). Diastolic blood pressure (DBP) was $\geq 90$ and $<110 \mathrm{mmHg}$, whereas systolic blood pressure (SBP) was $\geq 140$ and $<180 \mathrm{mmHg}$ in primary hypertension patients after a 1 -week placebo period. Exclusion criteria were as follows: i) Having such concurrent disease as secondary hypertension, severe hypertension, acute cerebrovascular accident during acute phase, white coat hypertension, congestive heart failure, unstable angina pectoris, acute myocardial infarction within 6 months, hyperkalemia and bilateral renal artery stenosis; and ii) other antihypertensive drugs were required. All the volunteers provided written consents prior to enrollment. All the subjects were determined by medical history, routine physical examinations, clinical laboratory testing and measuring the heart rate and blood pressure. After a 1-week washout period, baseline blood pressure values were recorded. All the subjects received a single oral dose of $5 \mathrm{mg} /$ day amlodipine (one 5-mg tablet; Pfizer Inc., Groton, CT, USA) with water $(200 \mathrm{ml})$ at 7-8 o'clock in the morning for 4 weeks. In total, $5 \mathrm{ml}$ blood was collected for each time point to measure serum trough concentrations of amlodipine at 4 weeks ( $\mathrm{C} 0$, steady-state trough blood concentration) and 4 weeks after the last dose at $2 \mathrm{~h}(\mathrm{C} 2$, plasma concentration at $2 \mathrm{~h}$ during absorption phase), $6 \mathrm{~h}$ (C6, plasma concentration at $6 \mathrm{~h}$ during peak phase) and $24 \mathrm{~h}$ (C24, plasma concentration at $24 \mathrm{~h}$ during elimination phase).

Pharmacokinetic analyses. The blood samples were immediately centrifuged at $1006.2 \mathrm{x}$ g for $10 \mathrm{~min}$. The plasma was separated and stored at $-20^{\circ} \mathrm{C}$ until further analyses. The plasma concentrations of amlodipine were measured by liquid chromatography/tandem mass spectrometry system with electrospray positive ionization mode (Waters, Milford, MA, USA). Light shading was enforced throughout the procedure. The valid range of analytes was $0.08192-20 \mu \mathrm{g} / \mathrm{l}$. The lower quantification limit of amlodipine was $0.3775 \mathrm{ng} / \mathrm{ml}$. There was an excellent linear relationship for the amlodipine calibration curve $\left(\mathrm{R}^{2}>0.9943\right)$. The accuracy range was $85-115 \%$. The intra- and inter-day variation coefficients of all the assays were $<15 \%$. The stability tests were suitable for analyzing a large volume of samples.

Pharmacodynamic analyses. Detected from the same patient arm with the calibrated Electronic Blood Pressure Monitor (HEM-7012; OMRON Inc., Nagoya, Japan), the blood pressures were recorded at $0 \mathrm{~h}$ of days 0 and 28 . The absolute variations in DBP and SBP from baseline were recorded after 4 weeks, and the antihypertensive efficiency was analyzed after 4 weeks.

Evaluation of therapeutic efficiency. Four weeks after sitting, DBP and SBP changes from baseline values $\triangle \triangle D B P$ and $\triangle \mathrm{SBP}$ ) were analyzed. After 4 weeks of antihypertensive therapy, the efficiency was evaluated. Antihypertensive effectiveness denoted sitting SBP/DBP as $<140 / 85 \mathrm{mmHg}$, sitting SBP decreasing by $20 \mathrm{mmHg}$ and/or sitting DBP decreasing by $10 \mathrm{mmHg}$.

Genotypic analyses. DNA was extracted from peripheral blood samples from 60 patients prior to the start of treatment. Genotypes for the POR A503V C $>$ T polymorphisms were determined by polymerase chain reaction-restriction fragment length polymorphism. The primers were TGGAGTACGAGACCAAGGCTG (forward) and GCTCCT GGATGAAGCCTA TG (reverse). $C Y P 3 A 4 * 1 G(\mathrm{G}>\mathrm{A})$ polymorphisms were analyzed by pyro sequencing. The primers were CACCCTGATGTCCAGCAGAAACT (forward) and AATAGA A AGAGATGAACCAGAGCC (reverse). $C Y P 3 A 5^{*} 3(\mathrm{~A}>\mathrm{G})$ and $M D R 1 C 3435 T$ were analyzed by direct automated DNA sequencing on ABI PRISM 3100 (Applied Biosystem, Foster City, CA, USA). The primers of $C Y P 3 A 5^{*} 3$ were CATGACTTAGTA GACAGATGA (forward) and GGTCCAAACAGGGAAGAGATA (reverse); MDR 1 C3435T: TGCTGGTCCTGAAGTTGATCTGTGAAC (forward) and AATAGAAAGCAGATGAACCAGAGCC (reverse).

Statistical analyses. Statistical analyses were performed by the SPSS software for windows (version 18.0; SPSS Inc., Chicago, IL, USA). The values are expressed as mean \pm standard 
Table I. Clinical characteristics of the 60 essential hypertensive patients.

\begin{tabular}{lc}
\hline Characteristics & Value \\
\hline Age, years & $57.67 \pm 7.88$ \\
Gender, $\mathrm{n}$ (male/female) & $31 / 29$ \\
Height, $\mathrm{cm}$ & $161.92 \pm 6.39$ \\
Weight, $\mathrm{kg}$ & $66.61 \pm 11.02$ \\
$\mathrm{BMI}, \mathrm{kg} / \mathrm{m}^{2}$ & $25.28 \pm 3.17$ \\
$\mathrm{SBP}, \mathrm{mmHg}$ & $152 \pm 10$ \\
$\mathrm{DBP}, \mathrm{mmHg}$ & $92 \pm 8$ \\
\hline
\end{tabular}

BMI, body mass index; SBP, systolic blood pressure; DBP, diastolic blood pressure.

deviation. $\mathrm{P}<0.05$ was considered to indicate a statistically significant difference. The measurement data of two groups were analyzed by t-test. Counting statistics of two groups was analyzed with $\chi^{2}$ and the pharmacokinetic parameters among different genotypic groups were compared with one-way analysis of variance.

\section{Results}

Patient characteristics. For the study, a total of 157 hypertensive patients were screened. Among them, 106 entered into the washout period and another 67 began the treatment period of amlodipine. A total of 60 patients completed the 4 -week treatment (Table I). There were 31 males and 29 females with an average age of $57.67 \pm 7.88$ years, an average height of $161.92 \pm 6.39 \mathrm{~cm}$, an average weight of $66.61 \pm 11.02 \mathrm{~kg}$ and an average body mass index of $25.28 \pm 3.17$. The common genetic polymorphisms of $C Y P 3 A 5, C Y P 3 A 4, P O R$ and MDRl were summarized in Table II. There were $32(53.3 \%)$ patients with the $C Y P 3 A 4 * 1$ GG genotype, $22(36.7 \%)$ with the $C Y P 3 A 4 * 1$ GA genotype, $6(10 \%)$ with the $C Y P 3 A 4 * 1 G$ AA genotype; $7(11.7 \%)$ with the $C Y P 3 A 5 * 3$ AA genotype, 24 (40\%) with the $C Y P 3 A 5 * 1$ AG genotype, $29(48.3 \%)$ with the $C Y P 3 A 5 * 3$ GG genotype; 29 (48.3\%) with the POR A503V CC genotype, 25 (41.7\%) with the POR A503V CT genotype, 6 (10\%) with the POR A503VTT genotype; and 30 (50\%) with the MDRI C3435T CC genotype, 24 (40\%) with the MDR1 C3435T CT genotype and $6(10 \%)$ with the MDRl C3435T TT genotype.

Association between gender and plasma concentration and efficacy of amlodipine. As shown in Table III, compared to males, the plasma concentrations of amlodipine in female hypertensive patients were significantly higher at each time point (C0, C2, C6 and $\mathrm{C} 24)(\mathrm{P}<0.05)$. However, no significant gender differences existed in antihypertensive efficacies $(\mathrm{P}>0.05)$.

Effects of CYP3A4*1G (G>A), CYP3A5*3 and POR A503V genotypes on plasma concentration and efficacy of amlodipine. As shown in Tables IV-VI, CYP3A4*1G $(\mathrm{G}>\mathrm{A})$, CYP3A5*3 $(\mathrm{A}>\mathrm{G})$ and POR A503V genotypes had no significant impact on the blood drug concentration and efficacy of amlodipine $(\mathrm{P}>0.05)$.
Table II. Frequency of the $C Y P 3 A 4 * 1 G, C Y P 3 A 5 * 3, P O R$ $A 503 \mathrm{~V}$ and MDRl C3435T genotypes in the 60 essential hypertensive patients.

\begin{tabular}{lcr}
\hline Gene & Genotypes & No. of subjects $(\%)$ \\
\hline CYP3A4*1G & GG & $32(53.3)$ \\
$C Y P 3 A 4^{*} 1 G$ & GA & $22(36.7)$ \\
$C Y P 3 A 4^{*} 1 G$ & AA & $6(10.0)$ \\
$C Y P 3 A 5^{*} 3$ & AA & $7(11.7)$ \\
$C Y P 3 A 5^{*} 3$ & AG & $24(40.0)$ \\
CYP3A5*3 & GG & $29(48.3)$ \\
POR A503V & CC & $29(48.3)$ \\
POR A503V & CT & $25(41.7)$ \\
POR A503V & TT & $6(10.0)$ \\
$M D R 1 C 3435 T$ & CC & $30(50.0)$ \\
$M D R 1 C 3435 T$ & CT & $24(40.0)$ \\
$M D R 1 C 3435 T$ & TT & $6(10.0)$ \\
\hline
\end{tabular}

CYP, cytochrome P450; POR, cytochrome P450 oxidoreductase; MDR1, multidrug resistance protein 1.

Table III. Difference of plasma concentration and effect of amlodipine in gender.

\begin{tabular}{lccc}
\hline & \multicolumn{2}{c}{ Gender } & \\
\cline { 2 - 3 } Variables & Male & Female & P-value \\
\hline $\mathrm{C}, \mathrm{ng} / \mathrm{ml}$ & & & \\
$\mathrm{C} 0$ & $5.83 \pm 2.51$ & $9.03 \pm 3.4$ & 0.012 \\
$\mathrm{C} 2$ & $7.41 \pm 2.73$ & $10.53 \pm 4.75$ & 0.013 \\
$\mathrm{C} 6$ & $8.58 \pm 3.03$ & $13.19 \pm 4.84$ & 0.004 \\
$\mathrm{C} 24$ & $5.91 \pm 2.93$ & $8.86 \pm 2.98$ & 0.003 \\
$\mathrm{BP}, \mathrm{mmHg}$ & & & \\
$\Delta \mathrm{SBP}$ & $15.87 \pm 9.3$ & $19.41 \pm 10.01$ & 0.161 \\
$\Delta \mathrm{DBP}$ & $6.39 \pm 5.79$ & $19.41 \pm 10.01$ & 0.276 \\
Efficiency, $\%$ & 51.61 & 55.17 & 0.782 \\
\hline
\end{tabular}

BP, blood pressure; SBP, systolic blood pressure; DBP, diastolic blood pressure.

Effect of MDR1 C3435T genotype on plasma concentration and efficacy of amlodipine. As shown in Table VII, the plasma drug concentrations at $\mathrm{C} 2$ and C6 in patients with the CT genotype of MDR1 C3435T were significantly higher than those with TT genotype $(\mathrm{P}=0.02, \mathrm{P}=0.03)$. The serum concentration at $\mathrm{C} 2$ was higher in the $\mathrm{CC}$ genotype compared to the TT genotype $(\mathrm{P}=0.05)$. The MDR1 C3435T genotype had no impact on the antihypertensive efficacy of amlodipine $(\mathrm{P}>0.05)$.

\section{Discussion}

As one of the third-generations of long-acting CCBs, amlodipine has been widely used as an antihypertensive drug. Amlodipine 
Table IV. Effect of the $C Y P 3 A 4 * 1 G$ genotype on amlodipine plasma concentration $(\mathrm{C})$ and efficacy.

\begin{tabular}{|c|c|c|c|c|c|c|c|}
\hline \multirow[b]{2}{*}{ Variables } & \multicolumn{3}{|c|}{ Genotype } & \multicolumn{4}{|c|}{ P-value } \\
\hline & GG $(n=32)$ & GA $(n=22)$ & $\mathrm{AA}(\mathrm{n}=6)$ & GG vs. GA & GG vs. AA & GA vs. AA & ANOVA \\
\hline \multicolumn{8}{|l|}{$\mathrm{C}, \mathrm{ng} / \mathrm{ml}$} \\
\hline $\mathrm{C} 0$ & $7.79 \pm 3.21$ & $6.86 \pm 3.51$ & $7.53 \pm 3.33$ & 0.597 & 0.774 & 0.663 & 0.838 \\
\hline $\mathrm{C} 2$ & $7.66 \pm 3.36$ & $8.31 \pm 3.41$ & $9.51 \pm 4.61$ & 0.682 & 0.357 & 0.312 & 0.437 \\
\hline C6 & $11.92 \pm 6.79$ & $10.44 \pm 4.86$ & $10.91 \pm 4.11$ & 0.860 & 0.737 & 0.745 & 0.893 \\
\hline $\mathrm{C} 24$ & $7.29 \pm 3.18$ & $7.04 \pm 3.35$ & $7.44 \pm 3.36$ & 0.937 & 0.936 & 0.796 & 0.966 \\
\hline \multicolumn{8}{|l|}{$\mathrm{BP}, \mathrm{mmHg}$} \\
\hline$\triangle \mathrm{SBP}$ & $16 \pm 10$ & $19 \pm 10$ & $22 \pm 5$ & 0.391 & 0.155 & 0.409 & 0.326 \\
\hline$\triangle \mathrm{DBP}$ & $6 \pm 6$ & $8 \pm 6$ & $10 \pm 4$ & 0.138 & 0.130 & 0.659 & 0.178 \\
\hline Efficiency, \% & 43.75 & 63.64 & 66.67 & 0.151 & 0.813 & 0.606 & 0.280 \\
\hline
\end{tabular}

CYP, cytochrome P450; ANOVA, analysis of variance; C0, C at 4 weeks; C2, 2 h after the last dose at 4 weeks; C6, 6 h; C24, 24 h; BP, blood pressure; SBP, systolic blood pressure; DBP, diastolic blood pressure.

Table V. Effect of the $C Y P 3 A 5 * 3$ genotype on amlodipine plasma concentration (C) and efficacy.

\begin{tabular}{|c|c|c|c|c|c|c|c|}
\hline \multirow[b]{2}{*}{ Variables } & \multicolumn{3}{|c|}{ Genotype } & \multicolumn{4}{|c|}{ P-value } \\
\hline & $\mathrm{AA}(\mathrm{n}=7)$ & $\mathrm{AG}(\mathrm{n}=24)$ & GG (n=29) & AA vs. AG & AA vs. GG & AG vs. GG & ANOVA \\
\hline \multicolumn{8}{|l|}{$\mathrm{C}, \mathrm{ng} / \mathrm{ml}$} \\
\hline $\mathrm{CO}$ & $4.82 \pm 2.05$ & $7.88 \pm 4.17$ & $6.77 \pm 2.92$ & 0.095 & 0.130 & 0.262 & 0.137 \\
\hline $\mathrm{C} 2$ & $6.70 \pm 2.93$ & $9.91 \pm 4.86$ & $8.57 \pm 3.38$ & 0.109 & 0.189 & 0.242 & 0.156 \\
\hline C6 & $7.81 \pm 4.27$ & $12.07 \pm 5.56$ & $10.35 \pm 3.54$ & 0.073 & 0.110 & 0.198 & 0.083 \\
\hline $\mathrm{C} 24$ & $4.99 \pm 2.97$ & $7.96 \pm 3.85$ & $6.10 \pm 3.16$ & 0.070 & 0.405 & 0.059 & 0.062 \\
\hline \multicolumn{8}{|l|}{$\mathrm{BP}, \mathrm{mmHg}$} \\
\hline$\triangle \mathrm{SBP}$ & $20 \pm 5$ & $19 \pm 10$ & $16 \pm 11$ & 0.695 & 0.169 & 0.332 & 0.483 \\
\hline$\triangle \mathrm{DBP}$ & $10 \pm 4$ & $8 \pm 7$ & $6 \pm 5$ & 0.440 & 0.096 & 0.355 & 0.290 \\
\hline Efficiency, \% & 85.71 & 50.00 & 48.28 & 0.092 & 0.074 & 0.901 & 0.187 \\
\hline
\end{tabular}

CYP, cytochrome P450; ANOVA, analysis of variance; C0, C at 4 weeks; C2, 2 h after the last dose at 4 weeks; C6, 6 h; C24, 24 h; BP, blood pressure; SBP, systolic blood pressure; DBP, diastolic blood pressure.

shows great individual differences in clinical applications. The present study indicates that there was no affect of the $C Y P 3 A 4$, CYP3A5 and POR gene genetic polymorphisms on the pharmacokinetics and pharmacodynamics of amlodipine. The genetic polymorphism of $M D R 1$ had an affect on the amlodipine pharmacokinetics, but there was no influence on its pharmacodynamics. Gender had an influence on the pharmacokinetics of amlodipine, but also affected the efficacy of amlodipine.

Amlodipine is mainly catalyzed by metabolism in the liver by cytochrome $\mathrm{P} 450$ family member $C Y P 3 A$. Bhatnagar et al (15) found that CYP3A4 (T16090C) had an influence on the curative effect of amlodipine, but CYP3A4 (-A392G) had no clear influence on the curative effect of amlodipine, in patients with early hypertensive renal disease. There was no influence of CYP3A4*1G on pharmacokinetics and pharmacodynamics of amlodipine in hypertensive patients.

The $C Y P 3 A 5^{*} 3$ locus mutation causes a lack of CYP3A5 enzyme activity. The present study found that the plasma concentrations of amlodipine in the $C Y P 3 A 5 * 3$ mutation genotype were higher compared to those of the wild genotypes. However, the differences had no statistical significance. The result may be associated with a small sample size.

The POR gene is the sole electronic donor for CYP enzyme metabolism and its coding gene has genetic polymorphism. Studies have shown that the genetic polymorphism of the $P O R$ gene is associated with the activity of CYP enzyme. The common mutations of $A 503 \mathrm{~V}$ in the $P O R$ gene affected the activity of CYP3A $(8,16,17)$. The genetic polymorphism of POR A503V affected CYP activity so as to influence the plasma concentration of amlodipine. However, the present study has not confirmed the effect of $P O R$ polymorphism on the plasma concentration of amlodipine due to a limitation of sample size.

Studies have shown that the gene polymorphism of $M D R 1$ directly affected the expression and function of P-gp. Investigators have found that the plasma concentration and area under the curve of amlodipine in the MDRI C3435T 
Table VI. Effect of the POR A503V genotype on amlodipine plasma concentration (C) and efficacy.

\begin{tabular}{|c|c|c|c|c|c|c|c|}
\hline \multirow[b]{2}{*}{ Variables } & \multicolumn{3}{|c|}{ Genotype } & \multicolumn{4}{|c|}{ P-value } \\
\hline & $\mathrm{CC}(\mathrm{n}=30)$ & $\mathrm{CT}(\mathrm{n}=24)$ & $\mathrm{TT}(\mathrm{n}=6)$ & $\mathrm{CC}$ vs. CT & CC vs. TT & CT vs. TT & ANOVA \\
\hline \multicolumn{8}{|l|}{$\mathrm{C}(\mathrm{ng} / \mathrm{ml})$} \\
\hline $\mathrm{CO}$ & $7.26 \pm 3.43$ & $7.43 \pm 3.49$ & $7.13 \pm 2.85$ & 0.87 & 0.93 & 0.85 & 0.98 \\
\hline $\mathrm{C} 2$ & $9.54 \pm 4.59$ & $8.55 \pm 3.85$ & $7.29 \pm 2.10$ & 1 & 0.25 & 0.45 & 0.42 \\
\hline C6 & $11.34 \pm 4.80$ & $10.3 \pm 4.67$ & $10.64 \pm 3.83$ & 0.43 & 0.74 & 0.87 & 0.72 \\
\hline $\mathrm{C} 24$ & $7.21 \pm 3.21$ & $7.37 \pm 3.60$ & $7.16 \pm 2.65$ & 0.79 & 0.85 & 0.99 & 0.96 \\
\hline \multicolumn{8}{|l|}{$\mathrm{BP}(\mathrm{mmHg})$} \\
\hline$\triangle \mathrm{SBP}$ & $19 \pm 10$ & $17 \pm 9$ & $15 \pm 8$ & 0.45 & 0.36 & 0.62 & 0.56 \\
\hline$\triangle \mathrm{DBP}$ & $8 \pm 6$ & $7 \pm 6$ & $6 \pm 5$ & 0.59 & 0.50 & 0.76 & 0.75 \\
\hline Efficiency, \% & 55.52 & 44.00 & 33.33 & 0.11 & 0.14 & 0.63 & 0.17 \\
\hline
\end{tabular}

POR, cytochrome P450 oxidoreductase; ANOVA, analysis of variance; C0, C at 4 weeks; C2, 2 h after the last dose at 4 weeks; C6, 6 h; C24, 24 h; BP, blood pressure; SBP, systolic blood pressure; DBP, diastolic blood pressure.

Table VII. Effect of the MDRl C3435T genotype on amlodipine plasma concentration (C) and efficacy.

\begin{tabular}{|c|c|c|c|c|c|c|c|}
\hline \multirow[b]{2}{*}{ Variables } & \multicolumn{3}{|c|}{ Genotype } & \multicolumn{4}{|c|}{ P-value } \\
\hline & $\mathrm{CC}(\mathrm{n}=30)$ & $\mathrm{CT}(\mathrm{n}=24)$ & $\mathrm{TT}(\mathrm{n}=6)$ & CC vs. CT & CC vs. TT & CT vs. TT & ANOVA \\
\hline \multicolumn{8}{|l|}{$\mathrm{C}(\mathrm{ng} / \mathrm{ml})$} \\
\hline $\mathrm{CO}$ & $7.40 \pm 3.38$ & $7.49 \pm 3.54$ & $5.70 \pm 1.59$ & 0.93 & 0.33 & 0.33 & 0.61 \\
\hline $\mathrm{C} 2$ & $9.13 \pm 4.79$ & $9.39 \pm 2.95$ & $5.74 \pm 3.63$ & 0.82 & 0.11 & 0.02 & 0.14 \\
\hline C6 & $11.26 \pm 4.78$ & $11.32 \pm 4.13$ & $6.96 \pm 4.43$ & 0.96 & 0.05 & 0.03 & 0.09 \\
\hline $\mathrm{C} 24$ & $7.35 \pm 3.43$ & $7.43 \pm 3.08$ & $6.20 \pm 3.86$ & 0.77 & 0.39 & 0.46 & 0.66 \\
\hline \multicolumn{8}{|l|}{$\mathrm{BP}(\mathrm{mmHg})$} \\
\hline$\triangle \mathrm{SBP}$ & $18 \pm 9$ & $18 \pm 10$ & $17 \pm 15$ & 0.97 & 0.86 & 0.85 & 0.96 \\
\hline$\triangle \mathrm{DBP}$ & $6 \pm 5$ & $8 \pm 6$ & $10 \pm 11$ & 0.13 & 0.46 & 0.78 & 0.25 \\
\hline Efficiency, \% & 50.00 & 54.17 & 66.67 & 0.76 & 0.46 & 0.58 & 0.75 \\
\hline
\end{tabular}

MDR1, multidrug resistance protein 1; ANOVA, analysis of variance; C0, C at 4 weeks; C2, $2 \mathrm{~h}$ after the last dose at 4 weeks; C6, 6 h; C24, $24 \mathrm{~h}$; BP, blood pressure; SBP, systolic blood pressure; DBP, diastolic blood pressure.

mutant allele carriers were lower than that of the wild-type in healthy subjects (16). The plasma concentration in the MDRI C3435T locus mutation homozygous TT genotype was lower compared to the genotypes of CC and CT $(\mathrm{P}<0.05)(16)$. The present study was consistent with previous studies in healthy subjects. The peak concentration in the TT genotype was lower than those of the wild genotypes, CC and CT $(\mathrm{P}<0.05)$. However, the MDR1 C3435T genotype had no significant effect on the efficacy.

Significant gender differences existed in the plasma drug concentrations in the present study. The plasma drug concentrations of females were significantly higher than those of males. This may be due to the fact that females tended to have smaller weights. However, the efficacy of amlodipine was not affected by gender or genetics. Therefore, it may also be due to fewer samples.

In conclusion, gender and the MDRl gene polymorphism may affect the plasma concentration of amlodipine in hypertensive patients in a Chinese Han population. However, there was no impact on the efficacy of amlodipine.

\section{Acknowledgements}

The present study was supported by the International Science \& Technology Cooperation Program of China (grant no. 2014DFA30900), National Scientific Foundation of China (grant nos. 81373476, 81301924 and 81302851) and Scientific Foundation of Hunan Province (grant nos. 11JJ2054 and 14JJ7016).

\section{References}

1. Park JY, Kim KA, Lee GS, et al: Randomized, open-label, two-period crossover comparison of the pharmacokinetic and pharmacodynamic properties of two amlodipine formulations in healthy adult male Korean subjects. Clin Ther 26: 715-723, 2004. 
2. Morgado M, Rolo S, Macedo AF, et al: Predictors of uncontrolled hypertension and antihypertensive medication nonadherence. J Cardiovasc Dis Res 1: 196-202, 2010

3. Li WW, Wallhagen MI and Froelicher ES: Hypertension control, predictors for medication adherence and gender differences in older Chinese immigrants. J Adv Nurs 61: 326-335, 2008.

4. Lamb RE1 and Bradford MJ: Controlling hypertension to reduce the risk of stroke. Prog Cardiovasc Nurs 17: 186-191, 2002.

5. Fogari R, Zoppi A, Derosa G, et al: Effect of valsartan addition to amlodipine on ankle oedema and subcutaneous tissue pressure in hypertensive patients. J Hum Hypertens 21: 220-224, 2007.

6. Philipp T, Smith TR, Glazer R, et al: Two multicenter, 8-week, randomized, double-blind, placebo-controlled, parallel-group studies evaluating the efficacy and tolerability of amlodipine and valsartan in combination and as monotherapy in adult patients with mild to moderate essential hypertension. Clin Ther 29: 563-580, 2007.

7. Midtvedt K, Hartmann A, Holdaas H and Fauchald P: Efficacy of nifedipine or lisinopril in the treatment of hypertension after renal transplantation: a double-blind randomised comparative trial. Clin Transplant 15: 426-431, 2001.

8. Gutierrez A, Grunau A, Paine M, et al: Electron transfer in human cytochrome P450 reductase. Biochem Soc Trans 31: 497-501, 2003.

9. Agrawal V, Huang N and Miller WL: Pharmacogenetics of P450 oxidoreductase: effect of sequence variants on activities of CYP1A2 and CYP2C19. Pharmacogenet Genomics 18: 569-576, 2008.

10. Wu L, Gu J, Cui H, et al: Transgenic mice with a hypomorphic NADPH-cytochrome P450 reductase gene: effects on development, reproduction, and microsomal cytochrome P450. J Pharmacol Exp Ther 312: 35-43, 2005.
11. Dobrinas M, Crettol S, Oneda B, et al: Contribution of CYP2B6 alleles in explaining extreme (S)-methadone plasma levels: a CYP2B6 gene resequencing study. Pharmacogenet Genomics 23: 84-93, 2013.

12. Frazier L, Turner ST, Schwartz GL, Chapman AB and Boerwinkle E: Multilocus effects of the renin-angiotensin-aldosterone system genes on blood pressure response to a thiazide diuretic. Pharmacogenomics J 4: 17-23, 2004.

13. Cai J, Huang Z, Yang G, et al: Comparing antihypertensive effect and plasma ciclosporin concentration between amlodipine and valsartan regimens in hypertensive renal transplant patients receiving ciclosporin therapy. Am J Cardiovasc Drugs 11: 401-409, 2011.

14. The Hypertension Prevention Guideline Revision Committee of China: The Chinese hypertension clinic guide 2005. Chinese Journal of Hypertension 13: 1-37, 2005.

15. Bhatnagar V, Garcia EP, O'Connor DT, et al: CYP3A4 and CYP3A5 polymorphisms and blood pressure response to amlodipine among African-American men and women with early hypertensive renal disease. Am J Nephrol 31: 95-103, 2010.

16. Arlt W: P450 oxidoreductase deficiency and Antley-Bixler syndrome. Rev Endocr Metab Disord 8: 301-307, 2007.

17. Fluck CE, Nicolo C and Pandey AV: Clinical, structural and functional implications of mutations and polymorphisms in human NADPH P450 oxidoreductase. Fundam Clin Pharmacol 21: 399-410, 2007. 